\title{
Investigation of Stress-concentration Factors in Tensile Strips
}

\author{
A photoelastic and numerical investigation has \\ been carried out for different ratios of hole diameter \\ to width of tensile strips
}

by P. E. van Riesen and R. M. E. J. Spiering

\begin{abstract}
A photoelastic and a numerical investigation has been carried out to determine the stress-concentration factors at the edge of a central circular hole in a tensile strip for different ratios of hole diameter to width of the strip. The photoelostic data and the numerical results indicate that the stress-concentration foctor at the minimum cross-sectional area tends to a value of two if the ratio of the hole diameter to the strip-width approaches a value of one.
\end{abstract}

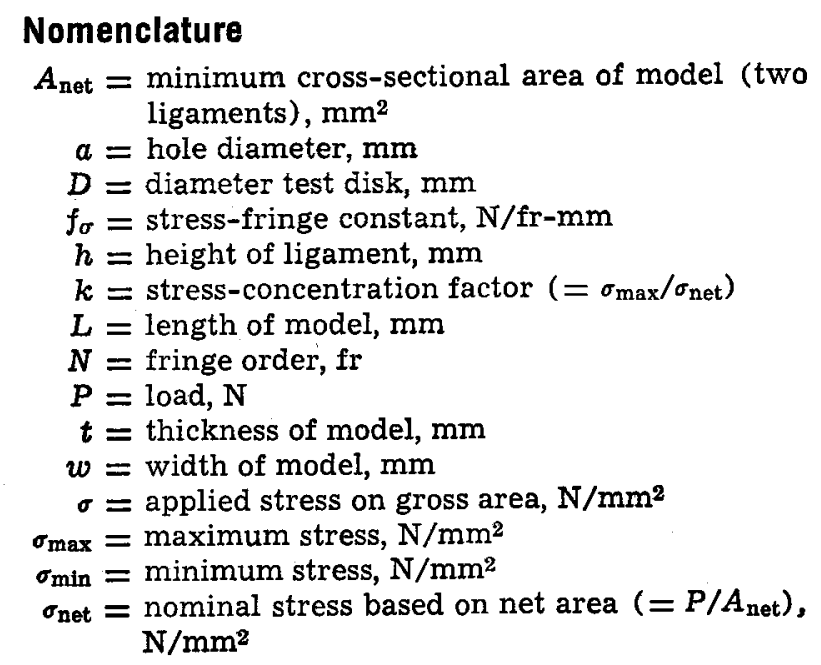

\section{Introduction}

A discussion has arisen about the stress-concentration factor $k$ at the edge of a central circular hole in a tensile strip when the ratio $a / w$ tends to one.1,2 In the photoelastic laboratory of the Applied Mechanics Group of the Twente University of Technology, an experimental investigation has been carried out to determine $k$ for values of $a / w$ in the range from 0.400

P. E. van Riesen and R. M. E. J. Spiering ate Cotworkers of the P. E. Van Riesen and R. M. E. J. Spieng Mechanical Engineering, Applied Mechanics Group, Department of Mechanical. Eng
Twente University of Technology, Enschede, Netherlands.

Original manuscript submitted: May 3, 1974. Revised version received: June $21,1974$. to 0.968. Simultaneously, a numerical analysis was executed by means of a computer program, based on a collocation method.

\section{Experimental Investigation}

A Sharples transmission polariscope, with quarterwave plates and a $360-\mathrm{mm}$ field was used. The light sources were a white (tungsten) source and a monochromatic one (sodium $5890 \AA$ ). In connection with the dimensions of the loadframe, the length $L$ of the model was fixed at $460 \mathrm{~mm}$ (see Fig. 1). In order to prevent any possible influence of the clamped-in ends of the strip on the stress distribution near the hole, the hole diameter was chosen to be $a=60 \mathrm{~mm}$. To reduce the width of the strip, the edges of the strip have been machined in twelve steps from the maximum width $w=150 \mathrm{~mm}(a / w=0.400)$ to a minimum of $w=62 \mathrm{~mm}(a / w=0.968)$. From an additional investigation, it became evident that a ligament thickness smaller than $1 \mathrm{~mm}$ gave rise to inaccurate results.

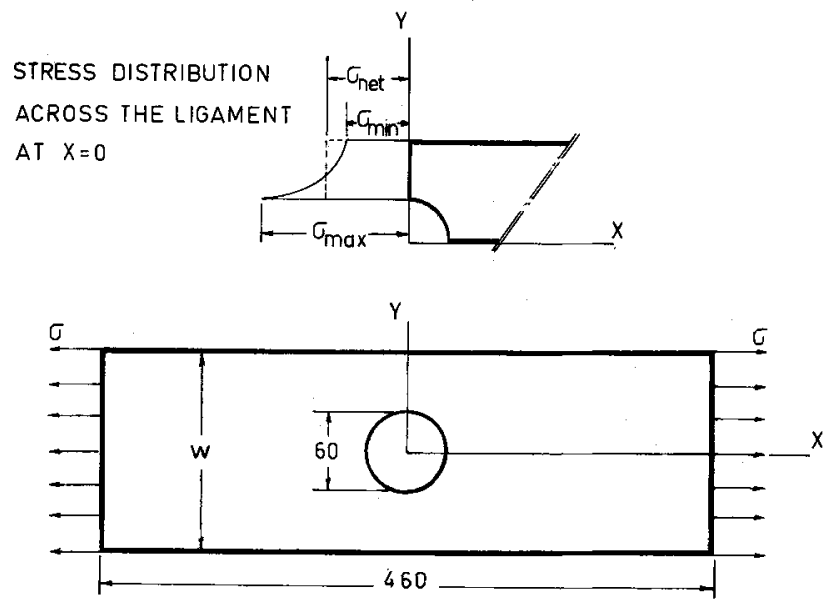

Fig. 1-Schematic of tensile strip 
At each value of $w$, the model was incrementally loaded at three levels in such a way that the maximum load produced six fringes at the minimum cross section. The two other load levels were a third and two thirds of the maximum load.

The loads were measured with a ring dynamometer. Araldite B was chosen as a model material because of its high sensitivity $\left(f_{\sigma}=11.7 \mathrm{~N} / \mathrm{fr}-\mathrm{mm}\right)$ and its good machinability.

The calibration has been carried out with a small cylindrical disk loaded by two radial opposite compressive forces $P$. These forces were increased from $100 \mathrm{~N}$ up to $500 \mathrm{~N}$ in steps of $100 \mathrm{~N}$.

The stress-fringe constant $f_{\sigma}$ was evaluated from the formula

$$
f_{\sigma}=\frac{8 P}{\pi N D}
$$

where $N$ is the fringe order at the center of the disk. The stress-fringe constant has been measured before and after the experiments. There was no significant difference between these two values.

From the fringe patterns of the loaded tensile strips dark- and light-field photographs were taken (see Figs. 2, 3, 4). The fringe orders across the ligament at $x=0$ have been plotted in Fig. 5 for $a / w=0.400$, 0.700 and 0.968 . For $a / w$ tending to one, the curves become linear, with the zero fringe order approaching the outside edge of the strip. Hence, the conclusion can be drawn that the stress-concentration factor tends to two when $a / w$ tends to one.

The values of $k$ also have been calculated from the formula:

$$
k=\frac{\sigma_{\max }}{\sigma_{\text {net }}}=N_{\max } \frac{f_{\sigma}}{t} \mid \frac{P}{A_{\text {net }}}
$$

In Fig. $6, k$ has been plotted against $a / w$.

\section{Numerical Investigation}

The numerical analysis has been done with a computer program developed at the Twente University of Technology. This program gives the numerical solution for stresses and displacements of plane elastostatic problems by a point-matching technique, as described by C. J. Hooke. ${ }^{3}$ This technique combines the collocation method with the method of least squares.

Equilibrium and compatibility conditions are satisfied in the region by a number of functions, each of them multiplied by an unknown coefficient. The number of boundary conditions can be taken larger than the number of unknown coefficients. In this case, the number of boundary conditions has been taken about twice as large as the number of coefficients.

An advantage of this method is that one can directly see from the difference between the numerical values of the prescribed and the calculated boundary conditions how well the prescribed boundary conditions are satisfied.

The results which are given in Fig. 6 indicate that $k$ tends to two for $a / w$ approaching the value of 0.968 . This is, in fact, the maximum value of $a / w$ at which the calculations gave reliable results.

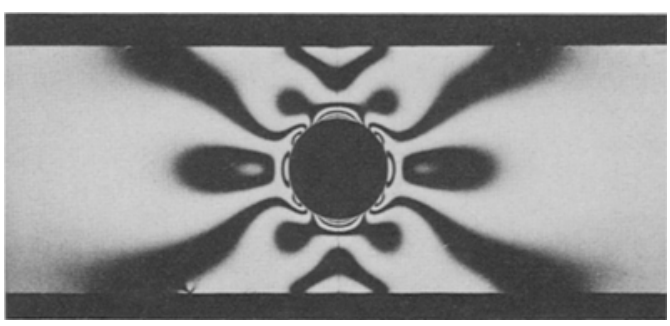

Fig. 2-Dark-field fringe pattern. a/w $=0.400$; $a=60 \mathrm{~mm} ; \mathrm{P}=2764 \mathrm{~N}$

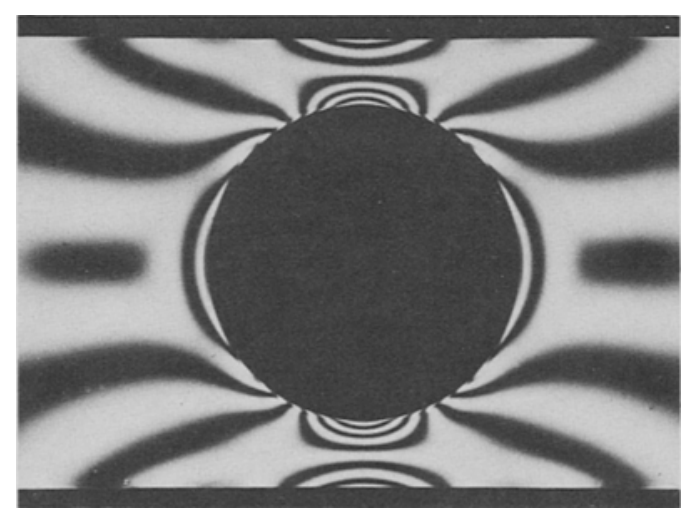

Fig. 3-Dark-field fringe pattern, $a / w=0.700$; $a=60 \mathrm{~mm} ; \mathrm{P}=824 \mathrm{~N}$

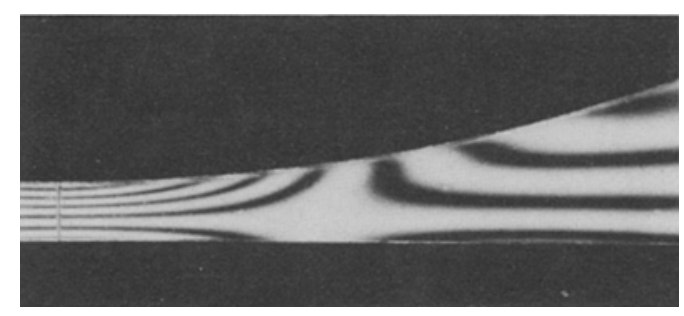

Fig. 4-Dark-field fringe pattern. $a / w=0.968$ $a=60 \mathrm{~mm} ; \mathrm{P}=75 \mathrm{~N}$

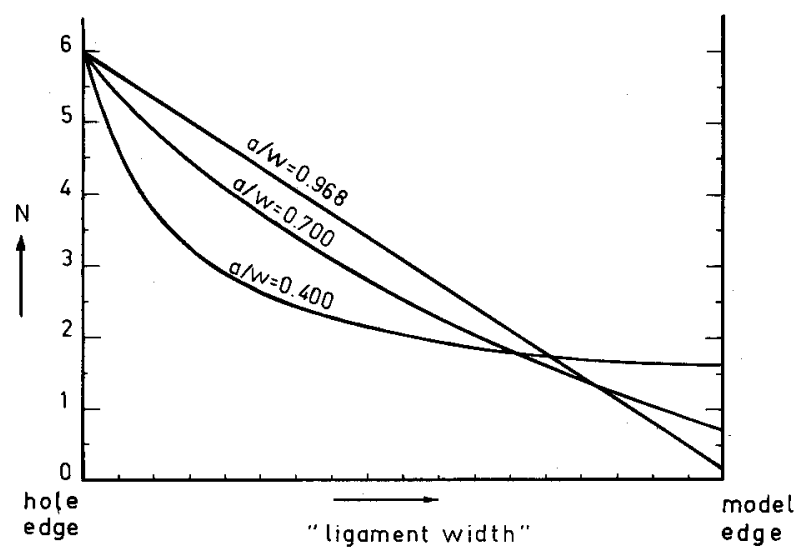

Fig. 5-Fringe orders across the ligament at $x=0$ 


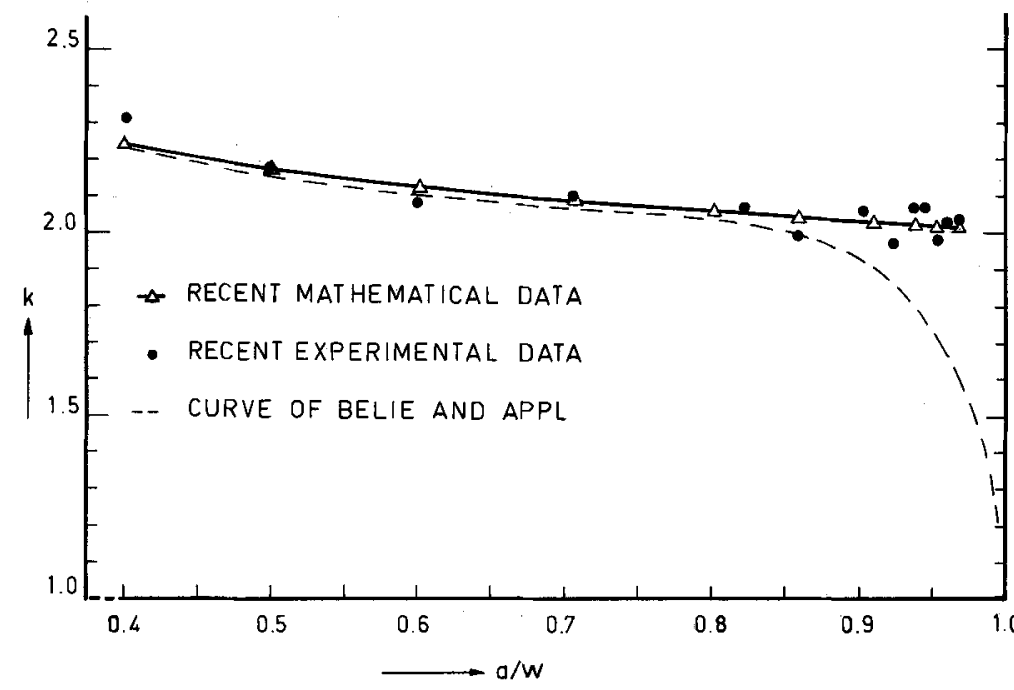

Fig. 7-Stress distributions for some values of $x(\mathrm{~mm})$

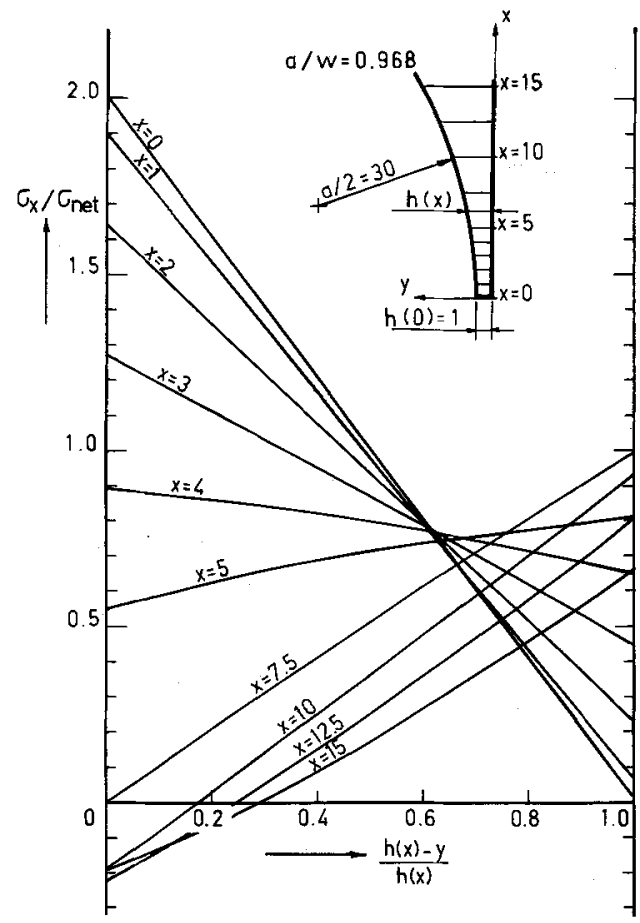

Fig. 6-Stress-concentration factors for tensile strips containing a central circular hole

TABLE 1 -COMPARISON OF DISPLACEMENT

\begin{tabular}{cc}
\hline$a / w$ & $\lambda$ \\
\hline 0.909 & 0.899 \\
0.938 & 0.928 \\
0.952 & 0.944 \\
0.968 & 0.961
\end{tabular}

$\lambda=$ ratio of the displacement at $x=0$ found by the point matching technique and found by Koiter.
With the program, it is also possible to compute stresses and/or displacements in inter-boundary and interior points. This made it possible to check the assumption made by Koiter ${ }^{4}$ of a linear stress distribution through the height $h$ of the ligament for $a / w$ approaching one.

From Fig. 7, where the computed stress distribution is given, it clearly appears that Koiter's assumption is admissible. Furthermore, the displacement at $x=$ 0 found from the formula stated by Koiter ${ }^{4}$ was compared with the computed displacement for different values of $a / w$ (see Table 1 ).

For $a / w=0.968$, the displacements of the center line of the ligament with respect to the displacement at $x=0$ were computed for a number of values of $x$ $\left(x_{\max }=20\right)$ and the results were compared with Koiters' formula. The differences between these two values were smaller than one percent.

\section{Conclusions}

For a number of values of $a / w$, the stress-concentration factor $k$ at the edge of a circular hole in a tensile strip has been measured by photoelastic means and calculated with a point-matching technique. The results are given in Fig. 6 . These results clearly indicate that the stress-concentration factor tends to two when $a / w$ tends to one.

The numerical results indicate that Koiters' assumption of a linear stress distribution for small ligament widths is admissible.

\section{References}

1. Belio, R. G. and Appl, F. J., "Stress Concentrations in Tensile Strips with Large Circular Holes," EXPERIMENTaL MEghanics, 12(4), 190-195 (1972).

2. Fuehring, H., "Stress Concentrations in Tensile Strips with Large Circular Holes-Discussion," EXPERIMENTal MEChanICs, 13(6), 255-256 (1973).

3. Hooke, C. I., "Numerical Solution of Plane Elastostatic Problems by Point-Matching," Strain Analysis, 3(2), 109-114 (1968).

4. Koiter, W. T., "An Elementary Solution of Two Stress-Concentration Problems in the Neighbourhood of a. Hole," Qtly. of Appl. Math., 15, 303 (1957). 